\title{
Claude Fleury, Ecrits de jeunesse. Tradition humaniste et liberté de l'esprit
}

Jean-Pierre Collinet

\section{(2) OpenEdition}

10 Journals

\section{Édition électronique}

URL : https://journals.openedition.org/studifrancesi/40602

DOI : 10.4000/studifrancesi.40602

ISSN : 2421-5856

Éditeur

Rosenberg \& Sellier

\section{Édition imprimée}

Date de publication : 1 juillet 2004

Pagination : 181-182

ISSN : 0039-2944

\section{Référence électronique}

Jean-Pierre Collinet, «Claude Fleury, Ecrits de jeunesse. Tradition humaniste et liberté de l'esprit », Studi Francesi [En ligne], 142 (XLVIII | I) | 2004, mis en ligne le 30 novembre 2015, consulté le 09 septembre 2021. URL : http://journals.openedition.org/studifrancesi/40602 ; DOI : https://doi.org/10.4000/ studifrancesi.40602

Ce document a été généré automatiquement le 9 septembre 2021.

\section{(c)}

Studi Francesi è distribuita con Licenza Creative Commons Attribuzione - Non commerciale - Non opere derivate 4.0 Internazionale. 


\title{
Claude Fleury, Ecrits de jeunesse. Tradition humaniste et liberté de l'esprit
}

\author{
Jean-Pierre Collinet
}

\section{RÉFÉRENCE}

CLAUDE FLEURY, Ecrits de jeunesse. Tradition humaniste et liberté de l'esprit. Edition établie et presentée par Noémi Hepp et Volker Kapp. Paris, Honoré Champion, 2003, («Sources classiques», $\left.\mathrm{n}^{\circ} 45\right)$.

1 A tous ceux qu'intéresse l'histoire littéraire du XVII ${ }^{\mathrm{e}}$ siècle français, les deux éditeurs de ce recueil offrent un régal de choix. Non qu'ils exhument, pour notre plaisir et l'enrichissement de nos connaissances, aucun document inédit. Ces deux dialogues portant pour titre «Si on doit citer dans les plaidoyers», même grossis et prolongés de «Remarques sur Homère» plus tardives, ainsi que d'un «Discours sur Platon», plus récent aussi, rédigés à l'âge de vingt-deux ans peuvent sembler ne pas peser bien lourd, comparés aux vingt volumes de l'«Histoire ecclésiastique» publiés plus tard par le même auteur, et surtout à son ouvrage novateur sur «Les Mœurs des Israélites», qui lui vaut le plus clair et le plus durable de sa notoriété, mais du moins on n'en ignorait pas l'existence. On continuait cependant à dédaigner ces opuscules dont le sujet en apparence trop ponctuel ne paraissait pas mériter qu'on y prête davantage d'attention, en dépit de la thèse soutenue en 1925 par l'abbé Gaquère sur Claude Fleury. Et l'on comprend que, dans sa «Bibliographie», A. Cioranescu n'ait pas cru bon de mentionner leur intitulé. Plus attentive et mieux inspirée, Noémi Hepp s'était aperçue très vite qu'ils valaient mieux que ce dédain. Elle aurait sans doute gardé pour elle cette précieuse découverte, sans l'insistance amicale de Volker Kapp et sa participation à l'entreprise. Remercions-les tous deux d'avoir tiré de l'ombre ce joyau si longtemps resté méconnu. 
2 Cosignée par eux, l'introduction liminaire, aussi dense que précise, élégamment écrite et si savamment informée qu'il est permis d'y voir un modèle du genre, met en lumière la qualité littéraire de ces textes, étonnante si l'on songe à la jeunesse du rédacteur, et cette maturité de la pensée, rare chez un écrivain à ses débuts. Ces quelques pages, à la rigueur, dispenseraient de poursuivre plus avant la lecture, tant elles disent tout. Mais, au contraire, elles donnent envie de se plonger, pour mieux se rendre compte par soimême, dans ces textes qu'on aurait crus plutôt rebutants. Et l'on n'y perd ni son temps, ni sa peine, car on est bientôt séduit par cet entretien entre gens de bonne compagnie, non moins plaisant que profond, en dépit de ce qu'il peut présenter, par endroits, d'encore un peu scolaire et qui lui donne en fin de compte un charme de plus.

Il met aux prises quatre interlocuteurs, dont trois, le cartésien Cordemoy, Michel Le Peletier, frère cadet du futur Contrôleur général des Finances, mais promis lui-même à de brillants emplois, et l'auteur, se déclarent partisans d'abolir l'usage, répandu parmi les avocats, de truffer leurs plaidoiries de citations extraites d'orateurs anciens, tels que Démosthène ou Cicéron, sans parler de tout ce qu'ils empruntent à des poètes et des prosateurs grecs ou latins, qu'ils allèguent plus pour témoigner de leur culture que pour les besoins de la cause qu'ils défendent. Ils en débattent avec Marillac, récemment installé comme Avocat général au Grand Conseil, et qui, sans se prononcer contre leur avis, accepte de tenir le rôle dévolu, dans les procédures de canonisation, à l'avocat du diable. La discussion se passe en septembre 1663 dans le parc de Rambouillet, après avoir commencé chez Le Peletier. Elle se déroule en deux journées consécutives, ce qui justifie la division en un couple de dialogues. La rédaction du premier ne s'achève pas avant le 17 mai 1664. Conversations réelles, comme inclinent à le penser les préfaciers? J'en douterais davantage, influencé, peut-être à tort, par ce que comporte de plus ou moins fictif, environ un lustre plus tard, la dispute qui s'élève, au cours d'une excursion à Versailles, sur le plaisir comparé du rire et des larmes, entre les «quatre amis» de La Fontaine dans sa «Psyché».

4 De la présente controverse, qui touche à de multiples centres d'intérêt, s'en dégagent, me semble-t-il, deux principaux. D'une part, en effet, l'œuvre du jeune Fleury s'inscrit dans le vaste mouvement qui vise à réformer, homogénéiser et rationaliser un appareil judiciaire où continue à se mêler au droit romain - justifiant le recours à la langue latine - le droit coutumier qui devrait la bannir des tribunaux, au profit de l'idiome vernaculaire, seul accessible à tous. À cet égard, le diptyque de Claude Fleury se situe quelque part à l'intersection des «Grands Jours d'Auvergne», tels que devait les relater, à peine une année plus tard Fléchier, et des «Plaideurs», la comédie de Racine, dont on ne lira plus, après avoir pris connaissance de ce que dit l'auteur de deux dialogues sur la place abusive prise dans le prétoire par les langues mortes, exactement dans la même perspective ce qui se rapporte, sur un mode résolument bouffon, au procès intenté contre le chien Citron.

5 Mais, d'un autre côté, la conversation, ici, ne se limite pas à ce qui se passe de blâmable ou de ridicule au Palais. Car nos quatre gens de justice, très cultivés, se doublent de fins lettrés, de sorte qu'ils ne se privent pas de se référer à la littérature, tant de l'Antiquité que de leur temps. Or on se trouve justement, à cette époque, dans un moment de transition capital, où le classicisme qui s'était imposé pendant le règne de Louis XIII cède la place à celui, moins pédantesquement doctrinal, du court apogée qu'il va bientôt atteindre au temps de Louis XIV, avant que ne couve, dès 1674, et n'éclate, en 1687, la Querelle des Anciens et des Modernes, où l'abbé Fleury tiendra sa place. Quand 
il rédige ses dialogues, il connaît Boileau, sait apprécier Molière; comme l'observent judicieusement les deux éditeurs de ce livre dans leurs notices et leur annotation, il semble appeler de ses vœux l'avènement, tout proche, d'un Racine et préparer la voie à la simplicité de son tragique, fondé sur l'intensité de la passion.

Enfin, lorsque nos jeunes contestataires s'insurgent et manifestent leur aspiration à s'émanciper d'un humanisme en passe de se scléroser dans une routine absurde, ne croirait-on pas entendre déjà La Fontaine (mêlé lui-même, quelque vingt ans auparavant, aux «Palatins» de «la Table ronde», jeunesse encore presque estudiantine, mais déjà sérieuse elle aussi, censée se familiariser avec les questions juridiques, tandis qu'il se préparait à devenir, sinon avocat, du moins détenteur d'offices dans les Eaux et Forêts, donc appelé pour une large part à des fonctions de judicature), lorsqu'il proclamera, dix ans après les dialogues de Fleury, dans son épitre à Huet, que son «imitation n'est point un esclavage», et qu'il entend ne prendre «que l'idée, et les tours, et les lois» que suivaient autrefois les plus autorisés parmi nos devanciers, donc respectueux de l'esprit, mais non asservi superstitieusement à la lettre, docile à l'exemple donné par l'Antiquité, sans renoncer pour autant à son indépendance et n'hésitant pas à déclarer: «Souvent à marcher seul j'ose me hasarder»? Fleury ne prête pas aux interlocuteurs de ses dialogues, dans un tout autre domaine, un langage très différent. Il ne s'agit pas là d'un trait particulier au poète, comme on pourrait le croire, mais d'une tendance plus générale qui tient une place prépondérante dans la mentalité de cette époque et son évolution. On souhaite alors, dans les milieux lettrés et cultivés, l'assouplissement d'un humanisme rendu trop rigide par les doctes, et la substitution au savoir livresque d'un goût mondain plus autonome et plus épris de clarté, de simplicité, d'organisation rationnelle et d'homogénéité. Le cartésianisme de Cordemoy va dans le même sens; il apporte à ces aspirations un puissant appui.

7 L'enjeu dépasse, on le voit, considérablement, les limites d'un réquisitoire contre les citations dans le prétoire, rarement indispensables, et plus nuisibles en général qu'efficaces pour la cause que l'on défend. Les Anciens s'en passaient le plus souvent: pourquoi ne pas les imiter, puisqu'on prétend se modeler sur leur exemple, plutôt que d'en prendre sur ce point le contre-pied? Argument quelque peu sophistique, mais plaisamment paradoxal; qui témoigne que l'argumentation proprement rhétorique développée dans ce double dialogue ne manque pas de saveur. Il arrive même qu'elle préfigure - en partièulier au sujet des passions - les idées exposées six années plus tard par Bernard Lamy dans son «Art de parler». Cet aspect de l'ouvrage ne doit cependant pas masquer ce qui reste d'essentiel et consiste dans les vues très lucides sur la mutation profonde qui s'observe à cette date dans un classicisme proche de son faîte, mais près aussi de déboucher tout droit sur une querelle entre les partisans des Anciens et ceux des Modernes dont les premières fêlures ne vont pas tarder à commencer d'apparaître.

8 Inutile de préciser que cette édition critique se recommande par le soin scrupuleux qu'ont apporté Noémi Hepp et Volker Kapp à l'établissement du texte. La copie qui sert de base à ce travail contenait quelques passages sur lesquels risquait d'achopper un lecteur d'aujourd'hui. Tous ont été corrigés avec soin et pertinence. Quelques-uns pouvaient ne pas l'exiger, car certaines tournures ou certains mots, sortis désormais de l'usage, ne l'étaient pas encore au temps de Fleury. Grâce au relevé des moindres détails, rien de plus facile que la reconstitution dans son état primitif du manuscrit qu'on doit au copiste. 
9 Cet impeccable travail, qui répare une longue injustice, comble une regrettable lacune et nous révèle de précieux documents, mérite de ne pas rester inaperçu. Que les éditeurs de ces opuscules soient chaleureusement félicités pour cette contribution vraiment magistrale aux études sur notre inépuisable Grand Siècle. 